\title{
Environnement bâti et santé
}

\author{
Booth $\mathrm{GL}^{1,2^{*}}$
}

\author{
Affiliations \\ ${ }^{1}$ Centre for Urban Health Solutions, Li Ka Shing Knowledge Institute et service d'endocrinologie, St. Michael's Hospital, Toronto (Ontario) \\ 2 Faculté de médecine; Institute of Health Policy, Management and Evaluation, Université de Toronto, Toronto (Ontario) \\ *Correspondance : boothg@smh.ca
}

Citation proposée : Booth GL. Environnement bâti et santé. Relevé des maladies transmissibles au Canada 2016;42:229-30. https://doi.org/10.14745/ccdr.v42i10a06f

\section{Contexte}

Depuis quelques décennies, nous assistons à un accroissement sans précédent du fardeau de l'obésité et du diabète à l'échelle mondiale. En Amérique du Nord et dans plusieurs autres régions du monde, cet accroissement coïncide avec les modifications apportées à l'aménagement urbain qui favorisent l'expansion des collectivités, où la dépendance automobile est marquée et où les lieux accessibles à pied sont rares. Dans leur quête d'approches populationnelles capables de freiner l'augmentation de l'obésité, les responsables de la santé publique se sont intéressés à l'environnement "bâti »-lequel comprend les éléments qui font partie de l'aménagement urbain et suburbain, comme les bâtiments, les rues, les parcs et les réseaux de transport - comme cible potentielle des politiques de santé publique.

\section{Objectif}

Déterminer si, au Canada, les quartiers urbains propices à la marche sont associés à de plus faibles niveaux d'embonpoint, d'obésité et de diabète que ceux qui sont moins adaptés à la marche, et étudier les répercussions, sur le taux de MTV dans les villes, des politiques qui encouragent un aménagement urbain favorable à la marche.

\section{Narratif}

Les quartiers construits après la Deuxième Guerre mondiale sont caractérisés par un fort étalement urbain, moins de liaisons entre les rues et des règlements de zonage qui séparent les terrains résidentiels des magasins locaux et d'autres installations. D'une manière générale, les résidents des zones suburbaines passent plus de temps dans une voiture, marchent moins, et utilisent moins le vélo et les transports en commun que ceux des zones urbaines, en plus d'être moins enclins que ces derniers à respecter les directives en matière $d^{\prime}$ activité physique (1-5). Non seulement les personnes qui vivent dans une communauté dépendante de l'automobile ont-elles moins d'occasions de faire de l'activité physique, mais elles sont plus susceptibles d'être exposées à de grandes quantités de polluants lorsqu'elles sont au volant (6).

Selon les résultats d'une étude d'observation canadienne et d'autres travaux de recherche, il est de plus en plus évident que les quartiers qui favorisent la marche et le vélo affichent des taux d'obésité et de diabète plus faibles que les autres (1,7-11). Le développement concerté des zones résidentielles et commerciales fait généralement augmenter l'indice de potentiel piétonnier. Les règlements de zonage qui permettent ce type de développement sont essentiels à l'aménagement de villes connectées et propices à la marche. D'autres mesures sont à même de favoriser l'activité physique, notamment le développement du transport en commun, ainsi que l'aménagement de parcs, d'espaces récréatifs, de sentiers et de pistes cyclables. Cependant, si le taux d'infections à transmission vectorielle continue de croître dans les zones urbaines, il faudra gérer le risque théorique accru de MTV causées par l'augmentation de l'exposition à l'extérieur. Les efforts visant à créer des espaces verts urbains propices à l'activité physique devront également prendre en compte les façons de réduire la possibilité que ces espaces deviennent des lieux favorables à la reproduction des moustiques et d'autres vecteurs.

\section{Conclusion}

La recherche semble indiquer qu'il existe un lien entre l'environnement bâti et les maladies liées à l'obésité.

L'aménagement des quartiers urbains est possiblement un autre élément à considérer face à l'émergence des MTV.

\section{Conflit d'intérêts}

Aucun.

\section{Références}

1. Creatore MI, Glazier RH, Moineddin R, Fazli GS, Johns A, Gozdyra G, Matheson FI, Kaufman-Shriqui V, Rosella LC, Manuel DG, Booth GL. Association of neighborhood walkability with change in overweight, obesity, and diabetes. JAMA. 2016;315(20):2211-20.

2. Sallis JF, Cerin E, Conway TL, Adams MA, Frank LD, Pratt M, Salvo D, Schipperijn J, Smith G, Cain KL, Davey R, Kerr J, Lai PC, Mitas J, Reis R, Sarmiento OL, Schofield G, Troelsen J, van Dyck D, De Bourdeaudhuij I, Owen N. Physical activity in relation to urban environments in 14 cities worldwide: a cross-sectional study. Lancet. 2016;387(10034):2207-17.

3. Hajna S, Ross NA, Brazeau AS, Bélisle P, Joseph L, Dasgupta K. Associations between neighbourhood walkability and 
daily steps in adults: a systematic review and meta-analysis. BMC Public Health. 2015;15:7684.

4. Sallis JF, Conway TL, Dillon LI, Frank LD, Adams MA, Cain $\mathrm{KL}$, Saelens BE. Environmental and demographic correlates of bicycling. Prev Med. 2013;57:456-60.

5. Carlson JA, Saelens BE, Kerr J, Schipperijn J, Conway TL, Frank LD, Chapman JE, Glanz K, Cain KL, Sallis JF. Association between neighborhood walkability and GPSmeasured walking, bicycling and vehicle time in adolescents. Health Place. 2015;32:1-7.

6. Health impacts of cars in London. London (UK): Greater London Authority. 2015 Sep. https://www.london.gov. uk/sites/default/files/health_impact_of_cars_in_londonsept_2015_final.pdf.

7. Booth GL, Creatore MI, Moineddin R, Gozdyra P, Weyman J, Matheson FI, Glazier RH. Unwalkable neighborhoods, poverty and the risk of diabetes among recent immigrants to Canada compared to long-term residents. Diabetes Care. 2013;36:302-8.

8. Glazier RH, Creatore MI, Weyman JT, Fazli G, Matheson Fl, Gozdyra P, Moineddin R, Kaufman-Shriqui K, Booth GL. Density, destinations or both? A comparison of measures of walkability in relation to transportation behaviors, obesity and diabetes in Toronto, Canada. PLoS One. 2014;9:e85295.

9. Wasfi RA, Dasgupta K, Orpana H, Ross NA. Neighborhood walkability and body mass index trajectories: Longitudinal study of Canadians. Am J Public Health. 2016;106(5):934-40.

10. Berry TR, Spence JC, Blanchard C, Cutumisu N, Edwards J, Nykiforuk C. Changes in BMI over 6 years: the role of demographic and neighborhood characteristics. Int J Obes (Lond). 2010;34(8):1275-83.

11. McCormack GR, Virk JS. Driving towards obesity: a systematized literature review on the association between motor vehicle travel time and distance and weight status in adults. Prev Med. 2014;66:49-55.

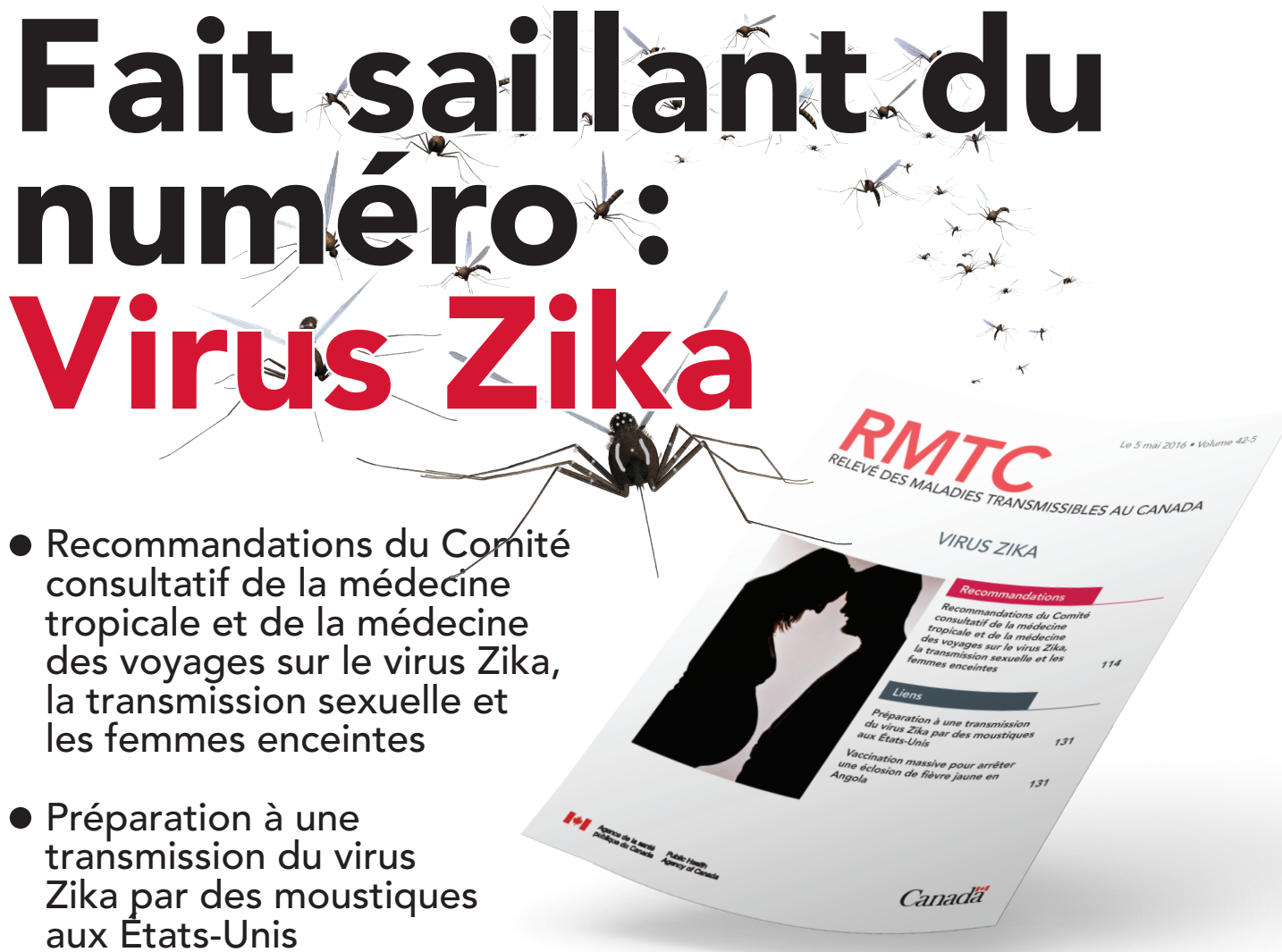

- Vaccination massive pour arrêter une éclosion de fièvre jaune en Angola

LIRE LE NUMÉRO : Recherche Web

\begin{tabular}{|l|l}
\hline RMTC+Zika & $\mathrm{Q}$
\end{tabular}

